\title{
Transición hacia la vida adulta en mujeres de sectores populares (Córdoba, Argentina)
}

\author{
Griselda Cardozo, Ph.D.
}

Universidad Católica de Córdoba, Argentina*

Ana Silvia González, Lic.

Universidad Católica de Córdoba, Argentina**

griseldacardozo656@hotmail.com

\section{Resumen (analítico)}

La transición hacia la adultez de los y las jóvenes de sectores populares en Argentina se tornó más prolongada y compleja. Introducir la perspectiva de género al problema obliga a interrogarse si, además, el ser mujer no constituye una dificultad adicional. Este trabajo analiza los modos en los que se produce la transición hacia la vida adulta en mujeres de sectores populares de Córdoba. Es un estudio cualitativo que utiliza como instrumento de recolección de datos el relato biográfico. Se trabajó con 12 mujeres entre 19 y 26 años que concurren a dos centros socioeducativos y laborales. Se concluye que la inserción al mundo adulto no se presenta del mismo modo. Se expresa en recorridos individuales -laborales y educativos - con problemáticas y tensiones que darían lugar a transiciones más o menos favorables.

\section{Palabras clave}

Joven desfavorecido/a, mujer, pobreza, biografía, transición a la vida activa, educación y empleo.

\section{Thesauro}

Tesauro de Ciencias Sociales de la Unesco.

\section{Para citar este artículo}

Cardozo, G., \& González, A. S. (2020). Transición hacia la vida adulta en mujeres de sectores populares (Córdoba, Argentina). Revista Latinoamericana de Ciencias Sociales, Niñez y Juventud, 18(3), 1-22. https://dx.doi.org/10.11600/1692715x.18303

Historial

Recibido: 14.01.2020

Aceptado: 29.04 .2020

Publicado: 13.08 .2020

\section{Información artículo}

Este artículo es parte del proyecto denominado «Procesos de subjetivación de los y las jóvenes en contextos de pobreza: trayectorias y proyectos de vida», financiado por la Universidad Católica de Córdoba e inscripto a la Secretaría de Investigación y Unidad Asociada al Conicet/Área en Ciencias Sociales y Humanidades/Facultad de Filosofía y Humanidades. Resolución Rectoral n. ${ }^{\circ} 991 / 16$. Realizado entre 01/03/2016 y 01/03/2019. Código del proyecto: $80020150200095 C C$. Investigación cualitativa con entrevistas. Gran Área de conocimiento: Ciencias Sociales. Área de conocimiento: Psicología. 


\section{Transition to adulthood among working-class women (Córdoba, Argentina)}

\section{Abstract (analytical)}

The transition to adulthood among working-class young people has become more prolonged and complex. Introducing the gender perspective to this problem leads to questioning whether being a woman represents an additional difficulty. This paper analyzes the ways in which the transition to adulthood among working-class young people of Córdoba (Argentina) occurs. This is a qualitative study that constructs biographies of the women and uses them as a data collection instrument. The research focused on 12 women between the ages of 19 and 26 who attend two different social labor educational centers. The transition to adulthood among young woman is not presented in the same manner. It is expressed in individual experiences - dealing with employment and education - that involve problems and tensions that affect the success of their transitions.

\section{Keywords}

Disadvantaged young people, women, poverty, biographies, transition to adulthood, education and employment.

\section{Transição para a vida adulta entre mulheres de setores populares (Córdoba, Argentina)}

Resumo (analítico)

A transição para a adultez entre os jovens e as jovens de setores populares na Argentina se tornou mais longa e complexa. Introduzir a perspectiva de gênero ao problema obriga a questionar se, além disso, o ser mulher não representa uma dificuldade adicional. Este trabalho analisa os diferentes caminhos de transição para a vida adulta entre mulheres de setores populares de Córdoba (Argentina). Trata-se de um estudo qualitativo que utiliza o relato biográfico como instrumento de coleta de dados. Foi realizado um trabalho com 12 mulheres de 19 a 26 anos de idade que frequentam dois centros socioeducativos e de preparação para o trabalho. A inserção no mundo adulto não se apresenta sempre do mesmo modo; ela percorre caminhos individuais - seja no âmbito do trabalho ou da educação - com problemáticas e tensões que levariam a transições mais ou menos favoráveis.

\section{Palavras-chave}

Jovem desfavorecido/a, mulher, miseria, biografia, transição à vida adulta, educação e emprego.

Información autoras

[*] Doctora en Psicología (Universidad Nacional de Córdoba). Posdoctorado en Ciencias Sociales, Niñez y Juventud, Centro de Estudios Avanzados del Cinde, Universidad de Manizales, Red Clacso. Licenciada en Psicología (Universidad Nacional de Córdoba). Profesora e investigadora en la Universidad Católica de Córdoba y Universidad Nacional de Córdoba. (iD) 0000-0002-1227-3255. Índice H5: 5. Correo electrónico: griseldacardozo656@hotmail.com

[**] Licenciada y profesora en Psicología. Especialista en Salud social y comunitaria de la Universidad Nacional de Córdoba. Profesora e investigadora de la Universidad Católica de Córdoba. (iD) 0000-0002-3634-3609. Correo electrónico: anasilviagonza@hotmail.com 


\section{Introducción}

$\mathrm{Z}_{\text {zado de formas diferentes según el contexto histórico y las teorías predominan- }}^{1 \text { proceso de transición de los las jenes a la vida adulta ha sido conceptuali- }}$ tes, por lo que se convirtió en un asunto de investigación y debate tanto en psicología como en otras disciplinas relacionadas.

Alrededor de los años sesenta, en las sociedades industrializadas como Estados Unidos, la mayoría de los y las jóvenes de 21 años entraba al mundo adulto a través de trayectorias vinculadas con la reproducción de las instituciones modernas en la vida cotidiana (la formación de una familia, el pasaje escolar y el ingreso al trabajo, etc.). Los y las jóvenes de aquella época crecían muy rápido y tenían que tomar decisiones importantes sobre su vida a una edad muy temprana (Arnett, 2015).

Actualmente, la vida de los y las jóvenes que transcurre entre los 18 y 29 años, etapa del ciclo vital a la que Arnett (2015) denominó «adultez emergente», es completamente diferente: tiene un desarrollo y características diferenciados de la adolescencia y la adultez. Se manifiesta como un fenómeno cultural en sociedades industrializadas a partir de los cambios sociodemográficos de las últimas décadas, tales como el incremento del acceso a la educación superior, el aumento de la fuerza laboral y la postergación de hitos como el matrimonio y la paternidad/maternidad (Arnett, 2008, 2015).

En esta línea, lo que interesa remarcar en este trabajo es lo que la literatura especializada desde la perspectiva de la sociología de las transiciones (Biggart et al., 2oo8; Casal et al., 2006; Miranda \& Corica, 2018) viene señalando: más que recorridos predecibles y claros en los que el proceso era entendido como el camino seguido por los y las jóvenes para llenar los «nichos» disponibles para ellos y ellas en el mercado laboral y en la sociedad, en el presente asistimos a un fenómeno en el que se entiende al proceso de transición a la adultez en función de trayectorias definidas como un camino a lo largo de la vida que puede variar y cambiar en dirección, grado y proporción (Elder, 2001). 
De esta manera, sobre todo en los países latinoamericanos, las transiciones se tornaron más prolongadas y complejas (Balarín et al., 2017; Miranda, 2016). Por un lado, a causa del impacto que provocaron las transformaciones económicas y sociales vinculadas a los cambios experimentados por los Estados de bienestar (Castel, 2012); y, por otro, dadas las condiciones propias del mundo globalizado que incentiva a los y las jóvenes a adoptar nuevos estilos de vida (Juárez \& Gayet, 2014).

Al respecto, Argentina no ha estado ajena a estos cambios sociodemográficos, los cuales se han complejizado para los y las jóvenes que provienen de los sectores populares que sufren pobreza y exclusión. Los datos señalan que en estos escenarios las privaciones se multiplican e inciden en sus biografías (Aisenson et al., 2015; Poy, 2018). A su vez, introducir la perspectiva de género obliga a preguntarnos si ser joven, pertenecer a sectores populares y, además, ser mujer, no constituye una dificultad adicional a la transición. El campo de estudio sobre juventud, educación y trabajo se encuentra en deuda con la perspectiva de género (Miranda \& Arancibia, 2017). En los últimos años, a pesar de los avances en las políticas sociales en materia de la incorporación de enfoques de género, se ha descuidado a las mujeres jóvenes (Trucco \& Ullmann, 2015); por ello, aún se sitúan como un colectivo marginado y desfavorecido, tanto en sus procesos de inserción laboral como en la posibilidad de construir carreras profesionales (Millenaar \& Jacinto, 2015).

En este sentido, la mayor participación laboral femenina en las últimas décadas no contribuyó a disminuir la segregación horizontal y vertical en el mercado de trabajo ni a reducir brechas salariales, todavía desfavorables para las mujeres. Dicha situación responde no solo a las características de demanda laboral, sino también a los mandatos culturales que señalan cuáles son los empleos asociados a los géneros (Arancibia \& Miranda, 2017). En los sectores populares estos imaginarios operan diferenciando la distribución de responsabilidades respecto del trabajo doméstico y extradoméstico, así como en las mayores entradas y salidas al empleo respondiendo a los períodos de maternidad. Así, las ofertas laborales para las jóvenes presentan características de mayor precariedad e inestabilidad (Millenaar, 2014).

Partiendo de esos debates, en este artículo nos propusimos presentar reflexiones y resultados de una investigación en la que se analizan los modos en los que se produce la transición hacia la adultez en jóvenes mujeres, de entre 18 y 26 años, de sectores populares de la ciudad de Córdoba (Argentina). Para tal fin, se exploran los cambios y continuidades en las trayectorias educativas y laborales, así como se indagan aspectos relativos a los eventos significativos que las delimitaron. Se consideran sus interrelaciones y 
confluencias para comprender cómo las jóvenes procuran organizar proyectos, enfrentar obstáculos, generar recursos y tomar decisiones en pos de un futuro.

Atendiendo a estos objetivos, se formularon los interrogantes que orientaron el trabajo: ¿qué características presenta la transición hacia la adultez en jóvenes mujeres de sectores populares?, ¿cuáles son las posibilidades o dificultades que enfrentan al transitar sus trayectorias educativas y laborales? y ¿qué decisiones toman ante el desafío que se les presenta en relación al futuro?

Un aporte valioso para el presente estudio lo constituye la perspectiva biográfica: es un marco referencial pertinente que toma al joven como sujeto histórico y protagonista de su vida, a partir de asumir sus propias elecciones y decisiones bajo determinaciones socioculturales (Roberti, 2017).

\section{Datos sobre escolaridad y empleo de las jóvenes de Córdoba}

A partir de la Ley n.․․ 26206 (Argentina, 2006) las políticas de inclusión educativa han ampliado la extensión de la educación obligatoria como derecho social y obligación del Estado hasta el nivel secundario. A pesar de ello, en los sectores populares continúa observándose una brecha entre la obligatoriedad de la escolarización y las posibilidades efectivas de acompañamiento que pueden realizar las familias en el ingreso a la escolaridad secundaria. En este sentido, el paso de la escuela primaria a la secundaria se identifica como el momento crucial en el que se produce el abandono escolar (Filmus, 2015). Por lo que siguen siendo los y las jóvenes de segmentos socio-ocupacionales desventajados quienes presentan mayores dificultades tanto para concluir la escuela como para continuar estudios superiores, situación que mella la igualdad de oportunidades y condiciona sus trayectorias posteriores (Poy, 2018), sobre todo en cuanto al ingreso a la vida laboral. El análisis que se realiza sobre la participación de las mujeres en relación a estos ámbitos permite comprender las desigualdades que se producen.

El reporte laboral del Ministerio de Producción y Trabajo (2018) advierte que las mujeres suelen tener una inserción laboral más precaria, lo que revela mayores tasas de desempleo, subempleo y empleo no registrado; y que suelen presentar tasas de menor actividad, lo que hace evidente el rol social que asume este colectivo, con una amplia dedicación al cuidado del hogar y las personas dependientes. En la misma línea, el Instituto Nacional de Estadística y Censos (2017) destaca entre los indicadores socioeconómicos 
para la población de 14 a 29 años perteneciente al Gran Córdoba en el cuarto trimestre del 2017, que la tasa de actividad arroja diferencias significativas entre varones $(54.3 \%$ y mujeres (37.9\%); que en la tasa de empleo para este grupo etario se ven desfavorecidas las mujeres (30.6\%) frente a los varones (49.1\%); que la tasa de desocupación para las mujeres indica una cifra de $19.2 \%$ contra $9.5 \%$ para los varones; y que el número de los que no estudian ni trabajan es de 49 263, integrando las mujeres el subgrupo más perjudicado ( 34162 mujeres que no estudian ni trabajan; un 22.3\% de la población femenina de esa edad).

\section{Método}

El proceso de investigación se realizó desde un enfoque cualitativo. Buscó centrarse en la narrativa de las jóvenes respecto a sus procesos de integración a la vida adulta; por ello, no se dirige a construir tipologías, sino a generar un proceso dialógico en el que se otorgue sentido a sus expresiones. Por tanto, esta perspectiva se ubica en una ontología materialista-dialéctica que permite abarcar el sistema relacional construido por el modo de conocimiento externo al sujeto, así como por los sentidos y significados que se constituyen sobre la vivencia de las relaciones objetivas de los actores sociales (Kornblit, 2004). En este marco, el estudio adoptó un diseño exploratorio-descriptivo y se recurrió a la implementación de herramientas cualitativas que favorecieron la elaboración de narrativas sobre los tópicos indagados, al mismo tiempo que contribuyó a la emergencia y el registro de aspectos no verbales.

\section{Participantes}

Para realizar el trabajo de campo se seleccionaron al azar dos instituciones, a saber: un centro socioeducativo y laboral de dependencia gubernamental y otro perteneciente a una comunidad religiosa.

Se conformó una muestra intencional con casos ricos para realizar un estudio en profundidad (Patton, 1990). Los criterios efectuados permitieron conformar una muestra de tipo: a) intensiva, al tratarse de casos de copiosa información, no necesariamente extremos o exóticos; b) caracterizada por la viabilidad y accesibilidad; c) representativa del fenómeno a estudiar (tener entre 18 y 26 años, residir en barrios de sectores populares).

La técnica de muestreo intencional y no probabilística utilizada fue la técnica de bola de nieve. El tamaño de la muestra, que se vincula con el grado de saturación teórica 
o conceptual, estuvo compuesta por 12 mujeres entre 19 y 26 años que concurren a los dos centros seleccionados (tabla 1 ).

\section{Tabla 1}

\section{Características sociodemográficas de las jóvenes}

\begin{tabular}{cclll}
\hline Nombre & Edad & Nivel de instrucción & \multicolumn{1}{c}{ Ocupación } & \multicolumn{1}{c}{ Estado civil/hijo } \\
\hline Jesica & 20 & Sec. incompleto & Estudiante & Soltera sin hijos \\
Soledad & 26 & Sec. completo & Empleada & Soltera sin hijos \\
Clara & 23 & Sec. completo & Empleada & Soltera sin hijos \\
María & 25 & Sec. incompleto & Desempleada & En pareja. Embarazada \\
Yanina & 22 & Sec. completo & Desempleada & Soltera sin hijos \\
Verónica & 22 & Sec. incompleto & Estudiante & En pareja sin hijos \\
Micaela & 22 & Sec. incompleto & Estudiante/Empleada & Separada un hijo \\
Rosalía & 20 & Sec. incompleto & Estudiante/Empleada & En pareja sin hijos \\
Laura & 19 & Sec. incompleto & Estudiante & Soltera sin hijos \\
Pamela & 19 & Sec. incompleto & Estudiante & Soltera sin hijos \\
Carmen & 20 & Sec. incompleto & Estudiante/Empleada & Soltera sin hijos \\
Sofía & 19 & Sec. incompleto & Estudiante & En pareja sin hijos \\
\hline
\end{tabular}

Nota. Sec. $=$ Secundario

El estudio se realizó de acuerdo con los requerimientos éticos de la Secretaría de Investigación en la que se enmarca el trabajo. Se tuvieron en cuenta los siguientes recaudos (Cornejo et al., 2008): se solicitó la autorización a las instituciones para efectuar las entrevistas y se modificó cualquier testimonio que permitiera identificar a las jóvenes. Al requerir su colaboración, se les leyó y entregó un consentimiento informado en el que se explicitó el marco institucional, los objetivos principales de la investigación, el carácter voluntario de la participación, la oportunidad de suspender el estudio cuando lo considerasen, las condiciones de anonimato y confidencialidad en el manejo de los datos recogidos. 


\section{Instrumento}

Para la construcción de los datos empíricos, se empleó el relato biográfico por ser una técnica que permite analizar la heterogeneidad de las temporalidades a partir de la co-construcción de los relatos alrededor de los acontecimientos que los sujetos señalan como significativos (Leclerc-Olive, 2009). En este sentido, la idea de trayectoria que aquí utilizamos se vincula a la de recorrido y no a una visión lineal del tiempo en sus cursos de vida (Casal et al., 2006), apuntando así al análisis de las transiciones y no de etapas fijas (Hareven, 1978).

\section{Procedimiento}

Los relatos fueron construidos a partir de cinco entrevistas en profundidad con la idea de ir recogiendo material referido a los diferentes campos y dimensiones de su guion (familia, grupo de pares, pareja, maternidad, escuela, barrio, religión, contextos o experiencias de riesgo o protección, duelos, proyectos a futuro). Estas fueron desgrabadas y transcritas para ser trabajadas en cada encuentro con las jóvenes. A partir del proceso de construcción de sus biografías, se fueron ordenando temporalmente los acontecimientos que delimitaron sus itinerarios vitales. El trabajo de campo se llevó a cabo entre el segundo semestre de 2017 y el primero de 2018.

Siguiendo la estrategia de la comparación constante de la teoría fundamentada, se construyó y codificó el corpus de entrevistas y relatos de manera simultánea. Se utilizó el software AtlasTi (versión 7.5.4). En diálogo con el estado del arte y el marco conceptual, se buscó aplicar los criterios de parsimonia y de alcance a los fines de identificar y desplegar analíticamente las categorías y proposiciones emergentes (Jones et al., 2004).

Se exponen las proposiciones emergentes en torno a las dos categorías centrales surgidas del análisis del corpus en las que confluyen las conexiones entre las dimensiones personales y estructurales constitutivas de las trayectorias que delimitan la transición hacia la adultez de las jóvenes: el lugar de la experiencia escolar en los itinerarios vitales y la experiencia laboral como medio para el logro de la autonomía e independencia. Alrededor de cada categoría se identificaron los acontecimientos y significaciones plasmados en el corpus de los relatos y entrevistas. 


\section{Resultados}

\section{El lugar de la experiencia escolar en los itinerarios vitales}

\section{El tránsito por la escuela entre la deserción y su finalización}

Las trayectorias escolares de las jóvenes se convirtieron en uno de los ejes centrales para el análisis de la transición hacia la adultez. Por trayectorias escolares se hace referencia al transcurrir de las jóvenes en condición de alumnas dentro del sistema educativo formal. Se distingue en ello las trayectorias teóricas (definidas por el sistema educativo dada su organización en un recorrido progresivo lineal) y las reales (se reconocen itinerarios frecuentes o probables que pueden o no coincidir con la trayectoria teórica) (Terigi, 2009).

En esta línea, comenzar a indagar acerca de sus recorridos por el sistema escolar implicó conectarse con los primeros recuerdos que se entrecruzan con eventos familiares afectados por carencias y que culminan en la transmisión de las desigualdades sociales relacionadas con la pobreza (condiciones laborales, de educación y lugar de residencia) que, tal como se presentan en sus relatos, se reproducen de generación en generación. Desde este escenario, a lo largo de las narrativas de las jóvenes se desplegaron diferentes situaciones sobre sus itinerarios. Todas completaron el nivel primario, aunque se presentan casos de repitencia. Esto constituye un indicio de que, en sus familias de origen, la escolarización básica fue considerada necesaria, a pesar de las dificultades estructurales. En relación con la escolaridad media: tres habían finalizado sus estudios secundarios, ocho los presentaban en curso y una los había abandonado. De este modo, pudieron identificarse tres modalidades de itinerarios escolares.

El primero dibuja un recorrido que se reconoce como la trayectoria esperada, en la que se encuentran aquellas jóvenes que finalizaron sus estudios, aunque no en los tiempos establecidos. En el caso de Yanina: los culmina con un período de interrupción de un año y teniendo que cambiar de escuela, al igual que Clara. En este grupo, que estaría en condiciones de iniciar estudios de nivel superior, se encuentran aisladas referencias respecto a la posibilidad de concretar este paso: «Cuando terminé no sabía bien qué carrera seguir; así que dije bueno, me tomo un respiro del secundario y después arranco. No he hecho ninguna carrera» (Relato de vida de Soledad, 2017).

El segundo recorrido se enmarca en una modalidad de trayectoria inconclusa para aquellas jóvenes que, por diferentes motivos, habían abandonado la escolaridad formal 
en el nivel secundario y en la actualidad deciden retomar bajo la modalidad que ofrecen estas instituciones (bachillerato acelerado). Desde la narrativa se advierte que, en una primera instancia, reproducen los recorridos de sus padres, quienes solo lograron finalizar la escolaridad primaria y en pocos casos comenzar la secundaria, pero sin poder finalizarla. En consecuencia, el clima educativo del hogar tiene un valor relevante en sus trayectorias escolares, aunque no puede determinarse por sí mismo como único motivo del rezago de las jóvenes.

Se detectan otros acontecimientos significativos que las condujeron a abandonar sus estudios, entre ellos, las historias familiares atravesadas por situaciones de violencia de género y un cúmulo de eventos conflictivos que permean sus cotidianidades. En esta línea, una joven refiere: «Fui al secundario, terminé segundo año y por esos problemas de violencia [en su familia] que me afectaron tuve que rendir libre. Entonces me quedé de año y decidí dejar y a los 18 empezar de nuevo» (Relato de vida de Pamela, 2018). De igual modo otra joven relata: «Dejé la escuela en segundo año cuando me fui de mi casa. Mi padrastro golpeó a mi mamá; yo la quise ayudar y me golpeó. Me fui a lo de mi abuela y no volví más» (Relato de vida de Carmen, 2018). Así, los testimonios muestran que el éxito o el fracaso de sus trayectorias escolares se dibujan al ritmo de los acontecimientos familiares vividos.

El caso extremo es el de una joven que vivió desde su infancia en instituciones estatales, luego de que sus padres la abandonaran y decide retomar sus estudios para mejorar sus posibilidades a futuro: «Vine al instituto cuando tenía 19 años. Necesitaba una banda de papeles que no tenía. Tuve que esperar todo ese año y, cuando ya tenía 2o, empecé. Ahora estoy en segundo» (Relato de vida de Verónica, 2017).

Además, para algunas de las jóvenes, el embarazo en plena adolescencia constituye un motivo por el cual deben interrumpir sus estudios: «Cuando yo tenía 15 años perdí un embarazo de tres meses fue un momento feo (...). Fue cuando me quedé de año» (Relato de vida de Sofía, 2018). En cambio, para otras jóvenes, el embarazo resulta un punto de inflexión que reconfigura sus trayectorias escolares:

Yo no quería estudiar, un año estudiaba, otro año no hacía nada y así, hasta que quedé embarazada a los 20 y dije «tengo que bajar a la tierra». Me anoté en el centro a distancia, pero no lo hacía; salía y me iba a trabajar y de ahí a la casa. Me volví a anotar en el semipresencial y tampoco lo hacés porque es casi lo mismo y este año me anoté en el presencial. Se me hace dura la mano con el bebé, hasta tener un trabajo estable se hace cuesta arriba. (Relato de vida de Micaela, 2017) 
Su historia refleja las dificultades que presentan la mayoría de las jóvenes mujeres al retomar el secundario, entre ellas, las de armonizar los requerimientos de la crianza de los hijos y las hijas (Balarin et al., 2017) con los propios del estudio y del trabajo. En estos casos, si bien la maternidad trae aparejada una serie de situaciones adversas, en relación con complementar estudio y trabajo, no altera por sí misma las condiciones de vida, sino que se suma a las condiciones estructurales heredadas. Para Micaela, la llegada de un hijo implica un modo de acceso a la adultez, un proyecto que le brinda una oportunidad diferente.

En contraposición, el éxito escolar en estos contextos se encuentra vinculado al apoyo que brindan los referentes significativos en el ámbito familiar o escolar a la hora de continuar los estudios. Una de las jóvenes recupera en su memoria:

Mi viejo me dijo que siguiera estudiando, que de otra forma no iba a tener un futuro. Porque mis hermanas dejaron la escuela, no conseguían trabajo y mi papá no podía mantenernos a todos. Mis padres no me dejan faltar a la escuela. Ellos me dicen «vos vas a la escuela que es tu prioridad». (Relato de vida de Jésica, 2017)

Otra joven señala la presencia de docentes dispuestos a comprometerse con la tarea y que apuestan a confiar en las posibilidades de sus estudiantes: «Más que alumnos-profesor, éramos amigos; si teníamos que sentarnos a conversar y tomar una Coca lo hacíamos. Había más comunicación, más diálogo, y te ayudaban» (Relato de vida de Micaela, 2017).

A modo de síntesis, se presentan en la tabla 2 los eventos significativos que se erigen como causas o motivos que delimitan las trayectorias inconclusas.

Por último, el recorrido escolar de las jóvenes se presenta como una trayectoria trunca para las que dejan definitivamente en el tramo de la escolaridad secundaria. A las causas de abandono reconocidas para las trayectorias inconclusas se suman factores que dan cuenta de los lugares subordinados que ocupan en general las mujeres en la sociedad. Señalan la pérdida súbita de ingresos por parte de sus familias de origen, la asunción de responsabilidades domésticas o laborales, lo que finalmente las conducen a renunciar a la escolaridad formal en la adolescencia:

Cuando tenía 5 años mi papá se fue. Se derrumbó la casa donde estábamos alquilando nosotros; después mi mamá pudo alquilar algo (...). Después mi mamá se desapareció, se había metido en el juego. Terminé tercer año, empecé cuarto y dejé a los 15 años. Ahora quiero terminar por el simple hecho de que diga [en el certificado] secundario completo, si consigo eso, me puede servir para todas las otras empresas. (Relato de vida de María, 2017) 


\section{Tabla 2}

\section{Eventos significativos}

\begin{tabular}{cccc}
\hline Nombre & $\begin{array}{c}\text { Año en curso al } \\
\text { momento de } \\
\text { abandono }\end{array}$ & Causas/motivos de abandono & Año en curso \\
\hline Jesica & $3^{\circ}$ & Repitencia & $5^{\circ}$ \\
Verónica & $1^{\circ}$ & Abandono/institucionalización & $2^{\circ}$ \\
Micaela & $5^{\circ}$ & Repitencia/embarazo & $5^{\circ}$ \\
Rosalía & $1^{\circ}$ & Problemas familiares & $3^{\circ}$ \\
Laura & $3^{\circ}$ & Repitencia & $3^{\circ}$ \\
Pamela & $2^{\circ}$ & Violencia familiar & $2^{\circ}$ \\
Carmen & $2^{\circ}$ & Problemas familiares/comienzo & $3^{\circ}$ \\
Sofía & $2^{\circ}$ & Repitenciayectoria laboral & $3^{\circ}$ \\
\hline
\end{tabular}

Su narrativa revela, al igual que otros trabajos realizados en nuestro país (Corica \& Otero, 2018), que las jóvenes ponderan, aún bajo ciertas dificultades, la continuidad educativa en vistas de la formación para el trabajo o bien para iniciar estudios superiores, por lo que las credenciales educativas siguen siendo una vía necesaria de transición hacia el mundo adulto.

\section{La experiencia laboral como medio para el logro de la}

\section{autonomía y la independencia}

\section{Entre las condiciones socioeconómicas heredadas y de género}

Si bien la participación de las mujeres en el mercado de trabajo y la distribución sexual del ingreso muestra una tendencia de largo plazo creciente, esto no significa que haya mejorado (Muñiz-Terra et al., 2013). Al analizar las experiencias laborales de las jóvenes se observa que los procesos de inserción se ven obstaculizados por el complejo entramado que se deriva de las condiciones sociales heredadas y de género.

La vulnerabilidad social de los hogares de los cuales provienen aparece como un factor que precipita su incorporación temprana al mercado laboral. Es clave la incidencia de la precariedad laboral del jefe o de la jefa de hogar o el recorte de sus ingresos monetarios. Así, la inestabilidad económica de toda la unidad familiar obliga a los hijos y las 
hijas a una pronta inserción en el mercado de trabajo y, en consecuencia, al abandono de sus estudios. Se observa que comienzan a trabajar tempranamente, entre los 13 y 15 años en el mejor de los casos, cuando no en plena infancia. Siempre en empleos precarios, en negro y con bajos salarios, sin beneficios ni protección alguna, que surgen ligados a la estricta supervivencia:

Pasamos momentos difíciles en la familia en los que la plata no alcanzaba. Cuando tenía 8 años a mi papá lo echan; él trabajaba en los colectivos. Tuvimos que salir a juntar cartón toda la noche con mis papás y mis hermanos. (Relato de vida de Clara, 2017)

Por otro lado, la problemática de la precarización laboral se asocia a factores familiares referidos a su composición, organización y dinámica. En las jóvenes, la fragilidad familiar (padres ausentes, violencia de género, alcoholismo) aparece como una de las causas del inicio en actividades informales. En estos casos se produce el paso de un hogar nuclear a uno monoparental de jefatura femenina. La madre deja las tareas domésticas y se vuelca al mercado laboral, mientras que la hija mayor abandona los estudios y se hace cargo de la organización doméstica, cuidado de hermanos menores, etc.

Mamá dijo que no nos iba a criar en un ambiente de drogadictos y alcohólicos, por eso se separaba. Yo me hice cargo de mis hermanas, pasé de ser una niña a grande, me hacía cargo de ellas, de sus horarios, del colegio, la comida. (Relato de vida de Micaela, 2017)

Bajo estas condiciones, asumen responsabilidades como una obligación y colaboración con sus madres a temprana edad. En otras circunstancias, estas actividades se complementan con un empleo doméstico temporario y esporádico en otras casas del barrio para sumar a los ingresos familiares; fundamentalmente, cuidado de niños, niñas y limpieza. Esto último parece ser una estrategia para compatibilizar las responsabilidades como proveedoras con la supervisión cotidiana de la marcha del hogar. En el caso de una joven, cuando su padre los abandona, comienza a colaborar con su madre, quien trabajaba cuidando a una persona mayor: «Ahí empecé a laburar cuidando nenes, como a los 12 o 13 años» (Relato de vida de María, 2017). Así, el acceso a la actividad remunerada implica una pérdida de derechos que nada tiene que ver con el disfrute de ventajas asociadas al abandono de la esfera doméstica.

Se advierte que en estos hogares se genera una reproducción de los modos más tradicionales de división sexual del trabajo, vinculados a los fuertes estereotipos de género. 
En estas familias, las mujeres se dedican a realizar trabajos extradomésticos antes de conformar pareja, y en algunas situaciones deben abandonarlos para dedicarse al cuidado familiar. En otras situaciones, la misma condición de ser mujeres las lleva a aprender desde niñas a defenderse solas, motivo por el cual aprenden labores típicamente masculinas: «Mi mamá siempre nos puso en la mente que, aunque seamos mujeres, no tenemos que esperar nada de nadie; todo tenemos que hacer: construcción, electricidad, planchar y cocinar» (Relato de vida de Micaela, 2017).

En cuanto a los estereotipos de género, las jóvenes plantean los mecanismos que operan en la división sexual del trabajo. Por un lado, con respecto a la valorización diferencial de espacios y actividades, se observa la persistencia de una segmentación de las ocupaciones por sexo (empleos típicamente masculinos y típicamente femeninos); las mujeres se orientan claramente hacia trabajos de servicio que requieren escasas habilidades, tal como lo demuestra la literatura (Micha \& Pereyra, 2019; Millenaar, 2014).

Trabajé en X (empresa comidas rápidas). Actualmente, hay dos días a la semana que trabajo en la casa de mi suegra, pero quiero entrar a la policía por el sueldo. Mi pareja me dice que la policía no es una carrera para la mujer. Me hizo un montón de cosas, me puso trabas que me hicieron retroceder; pero insistí y me anoté para aprender computación porque me va a servir para ingresar. (Relato de vida de Yanina, 2017)

En otras situaciones se observa un fuerte contraste en las actividades que comenzaron realizando como típicamente femeninas y el deseo de continuar en el futuro con actividades mejor remuneradas, pero en ámbitos laborales que en otros momentos se calificaban como típicamente masculinos:

Antes sí trabajaba de niñera cuidando a mis sobrinos, pero dejé de hacerlo porque no me gustan los niños (...); termino el secundario y quiero meterme en los agentes técnicos de la policía donde portás armas, vigilás las cámaras de seguridad y atendés llamadas de emergencia. Mi hermana y mi cuñado son policías y me hablaron de este programa que dura solo tres meses y se cobra un buen sueldo. (Relato de vida de Laura, 2018)

Así mismo, se evidencia la fuerte discriminación a la que deben enfrentarse, en lo que atañe a las condiciones laborales, tan solo por su condición de mujer. En general tienen oportunidades en empleos de menor calidad, con bajos salarios y en puestos de trabajos poco calificados que se asocian apocas horas de actividad laboral (heladerías, centros de atención telefónica, entre otras). De esta manera, además de las exclusiones y 
discriminaciones en las oportunidades de empleo que se ofrecen a las mujeres, las trayectorias femeninas se diferencian porque se ven orientadas por mandatos de género desde los cuales se asignan roles específicos a los sexos en relación al trabajo, aun para las jóvenes que logran terminar el nivel medio de educación (Millenaar \& Jacinto, 2015). En una de las jóvenes se suma la condición de maternidad. Estuvo trabajando tres años en un centro de atención telefónica, pero la despidieron en su cuarto mes de embarazo con una muy baja indemnización:

Tenía que quedarme 11 horas laburando ahí y no te daban dos mangos más, te seguían pagando el mísero sueldo y vos tenés que bancártela. Estuve media jornada en blanco y no me hacían los aportes como correspondía y me habían bajado de la obra social. (Relato de vida de María, 2017)

Paradójicamente, a pesar de que estas condiciones delimitan para las jóvenes trayectorias que se alejan de los parámetros esperables de mercado laboral formal, les permiten construir un oficio o acumular aprendizajes a partir de las experiencias:

Una vez que terminé el colegio empecé a trabajar en diferentes lados. Primero entré en un restaurante; ahí estuve un año y algo. Después estuve en venta de perfumes, en la calle. Luego estuve en un bar; después entré por temporada en un supermercado. (Relato de vida de Clara, 2017)

Lo que interesa aquí es remarcar que, más allá de que este recorrido se convierta en un aprendizaje, esta variación o desvinculación de los eslabones ocupacionales termina desdibujando las certidumbres en torno al trabajo y las formas de pasaje a la vida adulta (Miranda, 2016).

\section{La capacitación laboral como puerta hacia el futuro}

Tal como se pudo apreciar en los relatos de las jóvenes, la incorporación temprana al mercado de trabajo eleva la probabilidad de que deserten del sistema educativo, lo que restringe sus posibilidades futuras para obtener empleos de calidad. Como efecto, se observa una tensión entre las expectativas que tienen y las oportunidades que la sociedad les ofrece. Esto constituye un elemento central para orientar, moldear y modular el proceso de transición a la adultez (Miranda \& Corica, 2018).

En el caso de las jóvenes que no finalizaron aún el secundario, señalan esta situación como una deuda pendiente y reconocen la desigualdad que produce no contar con este 
título para conseguir trabajo. Frente a la dificultad palpable de inserción social, las jóvenes optan por construir sus propias trayectorias laborales a través de acciones y elecciones concretas:

Cuando yo estaba estudiando lo de la pelu [peluquería] fue justo cuando yo dejé el secundario a los 14 años. Me aferré mucho a eso entonces como que eso me fue tirando de a poquito afuera del pozo de donde estaba (...). Mi abuela me puso toda la peluquería, me la amobló toda, pero sé que tengo que estudiar para estar mejor. (Relato de vida de Carmen, 2018) A mí me encantó poder empezar a trabajar porque iba a poder tener mis cosas sin depender de nadie y, aparte, porque era chica cuando empecé; tenía 17 años y era difícil poder conseguir un trabajo sin tener los estudios terminados ni tener experiencia. (Relato de vida de Rosalía, 2018)

En este punto, comenzar la capacitación laboral se convierte en un medio para lograr mejores oportunidades a futuro y sortear las dificultades relacionadas con sus propias condiciones económicas y sociales, así como enfrentar las desigualdades de género. El desafío que se les presenta de mejorar sus trayectorias laborales, más allá del recorrido escolar alcanzado, constituye un hito significativo que se vive como un cambio de vida, tanto para las que finalizaron la escolaridad secundaria: «La capacitación me sirve para poner un microemprendimiento y obtener un sueldo para continuar estudiando» (Relato de vida de Clara, 2017); como para las que se encuentran aún en pleno recorrido: «Me sirve para aprender un oficio para obtener un dinero e ir acomodándome» (Relato de vida de Jésica, 2017); y para las que definitivamente abandonaron el sistema: «Yo siempre estaba por el tema del embarazo haciendo modificaciones a los jeans, un pantalón de embarazada te sale \$9oo y no daba pagar eso. Entonces cuando mi mamá me informó sobre esto [la capacitación laborall vine y enganché» (Relato de vida de María, 2017).

Finalmente, se observa que la transición a la adultez en las jóvenes se delimita a partir de itinerarios que escapan a las determinaciones lineales, si bien siguen subordinadas a sus situaciones biográficas, los capitales que heredan y los que logran acumular (Bourdieu, 200o), no necesariamente tienen un único final, sino que dependen de una diversidad de situaciones vitales que cambian el rumbo del futuro. En contextos como el estudiado, en el que el Estado es débil y las condiciones materiales son precarias, a mayor acumulación de barreras se generan transiciones más difíciles, por lo que el papel de la familia, la escuela y las redes sociales resulta crucial para generar transiciones más positivas (figura 1). 
Figura 1

Transición hacia la adultez: factores biográficos que inciden en las trayectorias escolares y laborales de las jóvenes

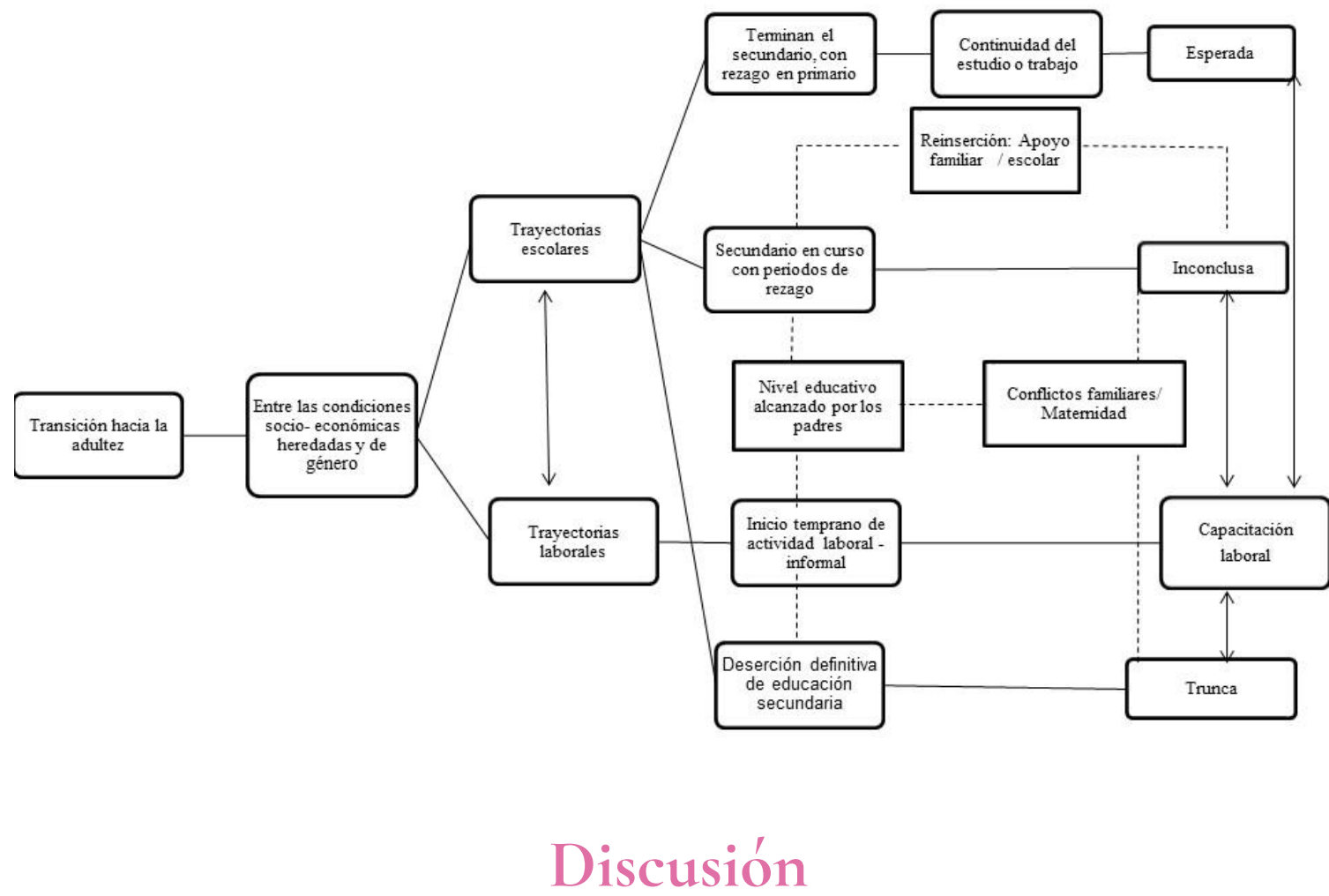

A partir del análisis de los relatos biográficos de las jóvenes de sectores populares que transitan la etapa del ciclo vital denominado adultez emergente, se detectaron algunos eventos y circunstancias que dan cuenta de sus modos de realizar este recorrido.

La decisión metodológica asumida habilitó reconstruir el encadenamiento de los acontecimientos que trazaron los itinerarios escolares y laborales, lo que permitió detectar que la inserción al mundo adulto en las jóvenes no se presenta del mismo modo: depende de los factores biográficos más objetivos con los que cuentan (los capitales educativos, por ejemplo) que se combinan con los factores subjetivos (valoraciones y disposiciones que tienen respecto del trabajo, las percepciones de su rol dentro de la familia, entre otros). En esta dirección se observa que tanto las opciones y las necesidades como las aspiraciones y los obstáculos se expresan en los recorridos individuales con problemáticas y tensiones que delimitan barreras derivadas de la ecuación de ser jóvenes, mujeres y pertenecer a sectores populares. Estas condiciones en sus diferentes combinacio- 
nes, sumadas a algunas oportunidades que les brinda el dispositivo de formación profesional, darían lugar a transiciones para algunas más y para otras menos favorables.

En este plano, y en contraposición con las jóvenes mujeres de sectores medios y altos, las trayectorias educativas y laborales difieren significativamente, ya que estas últimas tienen oportunidades de estudiar, de postergar su ingreso a las responsabilidades de la vida adulta y gozan de un período de menor exigencia en comparación con las jóvenes de sectores populares (Aparicio-Castillo, 2013; Poy, 2018). Se deriva de ello que el tránsito hacia el mundo adulto se produce de un modo cada vez más individualizado y en un contexto de gran desigualdad que pone en jaque el modelo normativo que establece la secuencia y los eventos legítimos durante la juventud, lo que trae aparejadas consecuencias en materia de autonomía, participación y acceso a los derechos sociales (Miranda \& Corica, 2018).

Surge como interrogante ante este escenario si las condiciones planteadas para estas jóvenes no terminarían generando un modo de transición denominado adultez forzada (Mora-Salas \& de Oliveira, 2014), al connotar en sí mismo un proceso acelerado rumbo a la vida adulta en la que las jóvenes se ven compelidas a asumir un rol activo a temprana edad (Sánchez-Galán, 2019).

En definitiva, los hallazgos del presente estudio ponen en tensión la idea de que la juventud es una etapa de transición hacia la vida adulta como período homogéneo. Posicionarse desde esta perspectiva constituiría un obstáculo epistemológico que impediría vislumbrar los complejos procesos que se ponen en juego al considerar otros factores como las condiciones socioeconómicas, culturales y de género. Atendiendo a esto, se tendrá en cuenta que la presente investigación implica el estudio de algunos casos, lo que constituye una limitación, por lo que sus resultados no podrían extenderse al campo general. No obstante, dichos casos aportan a la comprensión de la realidad local, así como contribuyen a pensar intervenciones desde lo social que avancen en disminuir las desigualdades existentes para este grupo.

En consecuencia, se convierte en un desafío a futuro la necesidad de profundizar el estudio en el colectivo de mujeres jóvenes ya que, si se llegaran a identificar otros factores que producen transiciones con rasgos de vulnerabilidad social, será posible diseñar políticas y programas (orientación vocacional laboral) que viabilicen este pasaje y mitiguen sus efectos, disminuyan la brecha de desigualdad existente con los varones, al tiempo que fomenten la promoción de expectativas y la generación de proyectos que favorezcan la inclusión de las jóvenes. 


\section{Referencias}

Aisenson, G., Legaspi, L., Valenzuela, V., Bailac, K. S., Czerniuk, R., Vidondo M., Virgili, N., Moulia, L., De Marco, M., \& Gómez-González, M. N. (2015). Temporalidad y configuración subjetiva: reflexiones acerca de los proyectos de vida de jóvenes en situaciones de alta vulnerabilidad social. Anuario de Investigaciones, 22(1), 83-92.

Aparicio-Castillo, P. Ch. (2013). Educar y trabajar en contextos de precariedad y desigualdad en América Latina: jóvenes en debate. Revista Latinoamericana de Ciencias Sociales, Niñez y Juventud, 11(2), 527-546.

Arancibia, M., \& Miranda, A. (2017). Modelos normativos, empleo y cuidados: las trayectorias de las mujeres jóvenes en el Gran Buenos Aires. En D. Beretta, E. Cozzi, M. Estévez, \& R. Trincheri (Comps.), Estudios sobre juventudes en Argentina V: juventudes en disputa: permeabilidad y tensiones entre investigaciones y políticas (pp. 186-196). Universidad Nacional de Rosario.

Argentina. (2006). Ley n. ${ }^{\circ} 26$ 206. Ley de Educación Nacional. Ministerio de Educación de la Nación.

Arnett, J. J. (2008). Adolescencia y adultez emergente: un enfoque cultural. Pearson.

Arnett, J. J. (2015). The Oxford handbook of emerging adulthood. Oxford University Press.

Balarin, M., Alcázar, L., Rodríguez, M. F., \& Glave, C. (2017). Transiciones inciertas: una mirada a los jóvenes de contextos urbanos vulnerables de Lima. Grade Group for the Analysis of Development. https://nbn-resolving.org/urn:nbn:de:0168-ssoar-56487-2

Biggart, A., Furlong, A., \& Cartmel, F. (2008). Biografías de elección y linealidad transicional: nueva conceptualización de las transiciones de la juventud moderna. En R. Bendit, M. Hanh, \& A. Miranda (Comps.), Los jóvenes y el futuro: procesos de inclusión social y patrones de vulnerabilidad en un mundo globalizado (pp. 49-71). Prometeo.

Bourdieu, P. (200o). Cuestiones de sociología. Istmo.

Casal, J., García, M., Merino, R., \& Quesada, M. (2006). Itinerarios y trayectorias: una perspectiva de la transición de la escuela al trabajo. Trayectorias, VIII(22), 9-20.

Castel, R. (2012). Prólogo. En G. Pérez-Soto \& M. Romero (Eds.), Futuros inciertos: informe sobre vulnerabilidad, precariedad y desafiliación de los jóvenes en el conurbano bonaerense (pp. 9-17). Instituto Torcuato Di Tella.

Corica, A., \& Otero, A. (2018). Transiciones juveniles: un análisis sobre el vínculo educación y trabajo de jóvenes egresados de la educación obligatoria argentina. Última Década, 26(48), 133-168. https://doi.org/10.4067/So718-22362018000100133 
Cornejo, M., Mendoza, F., \& Rojas, R. (2008). La investigación con relatos de vida: pistas y opciones del diseño metodológico. Psykhe, 17(1), 29-39. https://doi.org/10.4067/ So718-22282008000100004

Elder, G. H. (2001). Life course: Sociological aspects. En N. Smelser, \& P. Baltes (Eds.), International Encyclopedia of the Social and Behavioral Sciences. Elsevier.

Filmus, D. (2015). La universalización del secundario en América Latina: una meta necesaria para la inclusión social y la ampliación de derechos de los jóvenes. En A. Miranda (Ed.), Sociología de la educación y la transición al mundo del trabajo: Juventud, justicia y protección social en la Argentina contemporánea (pp. 103-116). Teseo.

Hareven, T. (Comp.) (1978). Transitions: The family and the life course in historical perspective. Academic Press.

Instituto Nacional de Estadística y Censos. (2017). Mercado de trabajo. Indicadores socioeconómicos (EPH): cuarto semestre de 2017. https://bit.ly/ziC4rxS

Jones, D., Manzelli, H., \& Pechey, M. (2004). La teoría fundamentada: su aplicación en una investigación sobre vida cotidiana con VIH/sida y con hepatitis C. En A. L. Kornblit (Coord.), Metodologías cualitativas en ciencias sociales: modelos y procedimientos de análisis (pp. 47-76). Biblos.

Juárez, F., \& Gayet, C. (2014). Transiciones a la vida adulta en países en desarrollo. Annual Review of Sociology, 4o(1), 1-18. https://doi.org/10.1146/annurevSOC-071312-145709

Kornblit, A. L. (2004). Metodologías cualitativas en ciencias sociales: modelos y procedimientos de análisis. Biblos.

Leclerc-Olive, M. (2009). Temporalidades de la experiencia: las biografías y sus acontecimientos. Iberóforum. Revista de Ciencias Sociales de la Universidad Iberoamericana, IV $(8), 1-39$.

Micha, A., \& Pereyra, F. (2019). La inserción laboral de las mujeres de sectores populares en Argentina: sobre características objetivas y vivencias subjetivas. Sociedade e Cultura, 22(1), 87-113. https://doi.org/10.5216/sec.v22i1.57887

Millenaar, V. (2014). Trayectorias de inserción laboral de mujeres jóvenes pobres: el lugar de los programas de formación profesional y sus abordajes de género. Trabajo y Sociedad, (22), 325-339.

Millenaar, V., \& Jacinto, C. (2015). Desigualdad social y género en las trayectorias laborales de jóvenes de sectores populares. El lugar de los dispositivos de inserción. En L. Mayer, D. Llanos, \& R. Unda-Lara (Comps.), Socialización escolar: procesos, experiencias 
y trayectos (pp.73-100). Abya Yala; Universidad Politécnica Salesiana; Cinde-Clacso. https://doi.org/10.2307/j.ctvtxw3k2.7

Ministerio de Producción y Trabajo. (2018). Provincia de Córdoba. Informe de diagnóstico laboral. https://bit.ly/33Sz7qe

Miranda, A. (2016). Transições juvenis, gerações sociais e processos de inclusão social na Argentina pós-neoliberal. Linhas Críticas, 22(47), 130-149. https://doi.org/10.26512/ 1c.v22i47.4797

Miranda, A., \& Arancibia, M. (2017). Repensar el vínculo entre la educación y el mundo del trabajo desde la perspectiva de género: reflexiones a partir de un estudio longitudinal en el Gran Buenos Aires. Archivos Analíticos de Políticas Educativas, (25), 1-19. https://doi.org/10.14507/epaa.25.2907

Miranda, A., \& Corica, A. (2018). Gramáticas de la juventud: reflexiones conceptuales a partir de estudios longitudinales en Argentina. En A. Corica, A. Freytes-Frey, \& A. Miranda (Comps.), Entre la educación y el trabajo: la construcción cotidiana de las desigualdades juveniles en América Latina (pp. 27-50). Clacso. https://doi.org/10.2307/ j.ctvfp62t8.4

Mora-Salas, M., \& de Oliveira, O. (2014). Los caminos de la vida: acumulación, reproducción o superación de las desventajas sociales en México. Revista Mexicana de Ciencias Políticas y Sociales, LIX(220), 81-115.

Muñiz-Terra, L., Roberti, E., Deleo, C., \& Hasicic, C. (2013). Trayectorias laborales en Argentina: una revisión de estudios cualitativos sobre mujeres y jóvenes. Revista Laboratorio, $14(25), 57-79$.

Patton, M. (1990). Qualitative evaluation and research methods. Sage.

Poy, S. (2018). Juventudes desiguales: oportunidades de integración social. Educa.

Roberti, E. (2017). Perspectivas sociológicas en el abordaje de las trayectorias: un análisis sobre los usos, significados y potencialidades de una aproximación controversial. Sociologías, 19(45), 300-335. https://doi.org/10.1590/15174522-019004513

Sánchez-Galán, F. (2019). Transición a la adultez en España antes y en la salida de la crisis económica: una comparación utilizando el análisis de entropía. Empiria. Revista de Metodología de Ciencias Sociales, (43), 117-136. https://doi.org/10.5944/ empiria.43.2019.24301

Terigi, F. (2009). Los cambios en los formatos de la escuela secundaria Argentina: por qué son necesarios, por qué son tan difíciles. Revista Propuesta Educativa, 1(29), 63-71. Trucco, D., \& Ullmann, H. (Eds.) (2015). Juventud: realidades y retos para un desarrollo con igualdad. Comisión Económica para América Latina y el Caribe. https://bit.ly/3gS 5 IQP 